\title{
Odontohypophosphatasia - A Case Report
}

\author{
Balaji Manohar, Lalit Mathur, Rajesh Pillai, Neema Shetty, Aman Bhatia and Aditi Mathur*
}

Department of Periodontics Pacific Dental College \& Hospital Debari, Udaipur, India

\begin{abstract}
Hypophosphatasia is a rare disease characterized by low levels of tissue non-specific alkaline phosphatase. It is characterized by defective bone and teeth mineralization. The symptoms are highly variable which ranges from stillbirth with defective mineralization of bone to early loss of tooth. The severity of disease is not directly related to serum alkaline phosphate levels. Some cases present with premature loss of only deciduous teeth without any bone disease. This condition is known as odontohypophosphatasia.
\end{abstract}

Here we report a case of 15 year old female who presented only dental findings of hypophosphatasia but had no appreciable bone defects seen.

Keywords: Odontohypophosphatasia; Alkaline phosphatase; TNSALP gene

\section{Introduction}

Hypophosphatasia was first described in 1948 by John Campbell Rathbun in an infant who died in infancy with severe rickets and seizure; and was found to have had low level of alkaline phosphatase [1].

Hypophosphatasia is a rare metabolic bone disease that is characterized by deficient activity of tissue-nonspecific alkaline phosphatase (TNSALP) [2]. This deficient activity of TNSALP has been attributed to mutations in TNSALP gene which causes significant changes in the structure and functions of TNSALP [3].

Hypophosphatasia is classified into different clinical types as followed [4]:
a. Perinatal
b. Infantile
c. Childhood
d. Adult
e. Odontohypophosphatasia and
f. Pseudohypophosphatasia.

Perinatal hypophosphatasia has the most severe manifestations. It is usually diagnosed at birth and infant rarely survives for more than few hours. Death occurs due to respiratory failure. Marked hypocalcification of skeletal structures is observed.

In infantile hypophosphatasia babies appear normal upto the age of 6 months but after this they begin to show a failure in growth. Vomiting and hypotonia may develop. Skeletal malformations like shortened, bowed limbs may be seen. Radiographs show a markedly reduced degree of ossification with preponderance of hypomineralized osteoid. These infants survive and premature shedding of deciduous teeth is often seen.

Childhood hypophosphatasia is detected at a later age with a consistent feature of premature loss of primary teeth without evidence of a significant inflammatory response. Teeth may show enlarged pulp chamber and significant degree of alveolar bone loss may be seen. Open fontanelles with premature fusion of cranial sutures may be seen. Affected patients have short stature, bowed legs and waddling gait. Radiographically, skull has an appearance of beaten copper and shows uniformly spaced, poorly defined, small radiolucency.

Adult form is mild with patients having history of premature loss of deciduous and permanent dentition and many patients are edentulous. Stress fractures involving the metatarsal bones may be a presenting sign.

Pseudohypophosphatasia is a disease resembling classic hypophosphatasia but normal serum alkaline phosphatase. There is osteopathy of long bones and skull, premature loss of deciduous teeth, hypotonia, hypercalcemia and phosphoethanolaminuria.

Odontohypophosphatasia is characterized by premature exfoliation of primary teeth and severe dental caries, often not associated with abnormalities of the skeletal system [5]. The anterior deciduous teeth are more likely to be affected and the most frequently lost. Radiographs show reduced alveolar bone and enlarged pulp chambers and root canals.

\section{Case Report}

A 15 year old female patient reported to the Department of Periodontics, Pacific Dental College and Hospital, Udaipur with a complaint of exfoliation of teeth soon after their eruption.

After eruption both primary and permanent teeth became carious and mobile, which led to gradual loss of teeth. There was no history of trauma. On recording the medical history it was noted that she had vomiting, chronic diarrhoea and weakness at the age of $2^{1 / 2}-3$ years, for which she was treated by blood transfusion 2-3 times. Calcium supplements were also given. But, she could not produce any documented evidence for the same. The patient was the youngest child in a family of three children. Parents, siblings and grandparents were healthy and did not show any signs of unusual tooth exfoliation. No bony deformity was detected.

Extraoral examination for abnormalities in eyes, temporomandibular joint, lymph nodes and presence of any swellings were non contributory.

*Corresponding author: Dr Aditi Mathur, Post graduate student, Department of Periodontics, Pacific Dental College \& Hospital, Debari, Udaipur, India; Tel: 09929826446; E-mail: draditimathur@gmail.com

Received October 07, 2010; Accepted October 21, 2010; Published October 23 2010

Citation: Manohar B, Mathur L, Pillai R, Shetty N, Bhatia A, et al. (2010) Odontohypophosphatasia - A Case Report. J Carcinogene Mutagene 1:105. doi:10.4172/2157-2518.1000105

Copyright: (c) 2010 Manohar B, et al. This is an open-access article distributed under the terms of the Creative Commons Attribution License, which permits unrestricted use, distribution, and reproduction in any medium, provided the original author and source are credited. 
Citation: Manohar B, Mathur L, Pillai R, Shetty N, Bhatia A, et al. (2010) Odontohypophosphatasia - A Case Report. J Carcinogene Mutagene 1:105. doi:10.4172/2157-2518.1000105

Page 2 of 3

Intraoral examination showed presence of first and second permanent molars in all quadrants and the maxillary right central incisor (Figure 1 and Figure 2). Retained roots of deciduous maxillary right first molar, maxillary left canine and mandibular right lateral incisor were present (Figure 3).

Patient had poor oral hygiene (Figure 4). Gingiva was red in colour, soft and edematous in consistency with increase in size of marginal and attached gingiva. There was grade II gingival recession and grade II mobility in relation to first molars in the second and
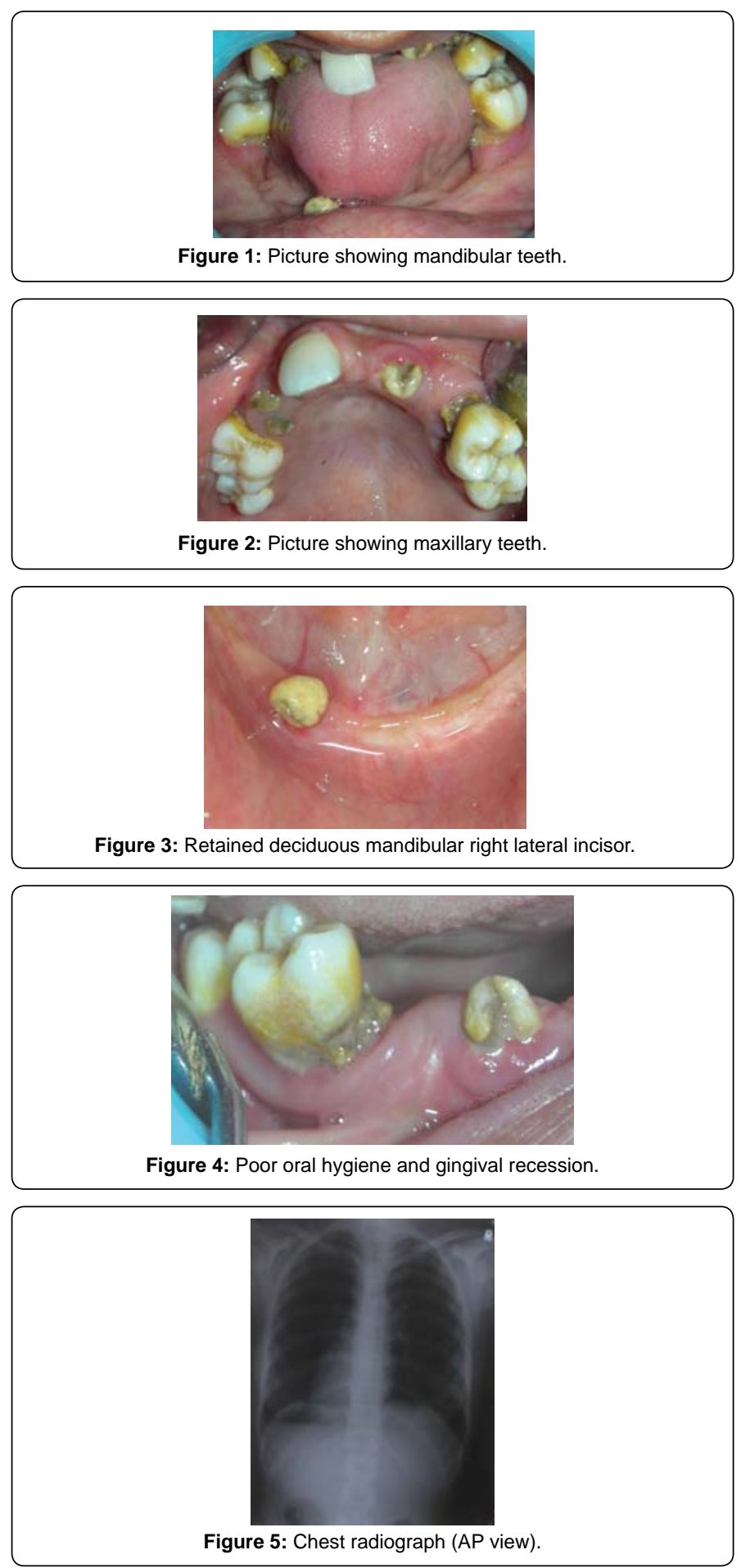
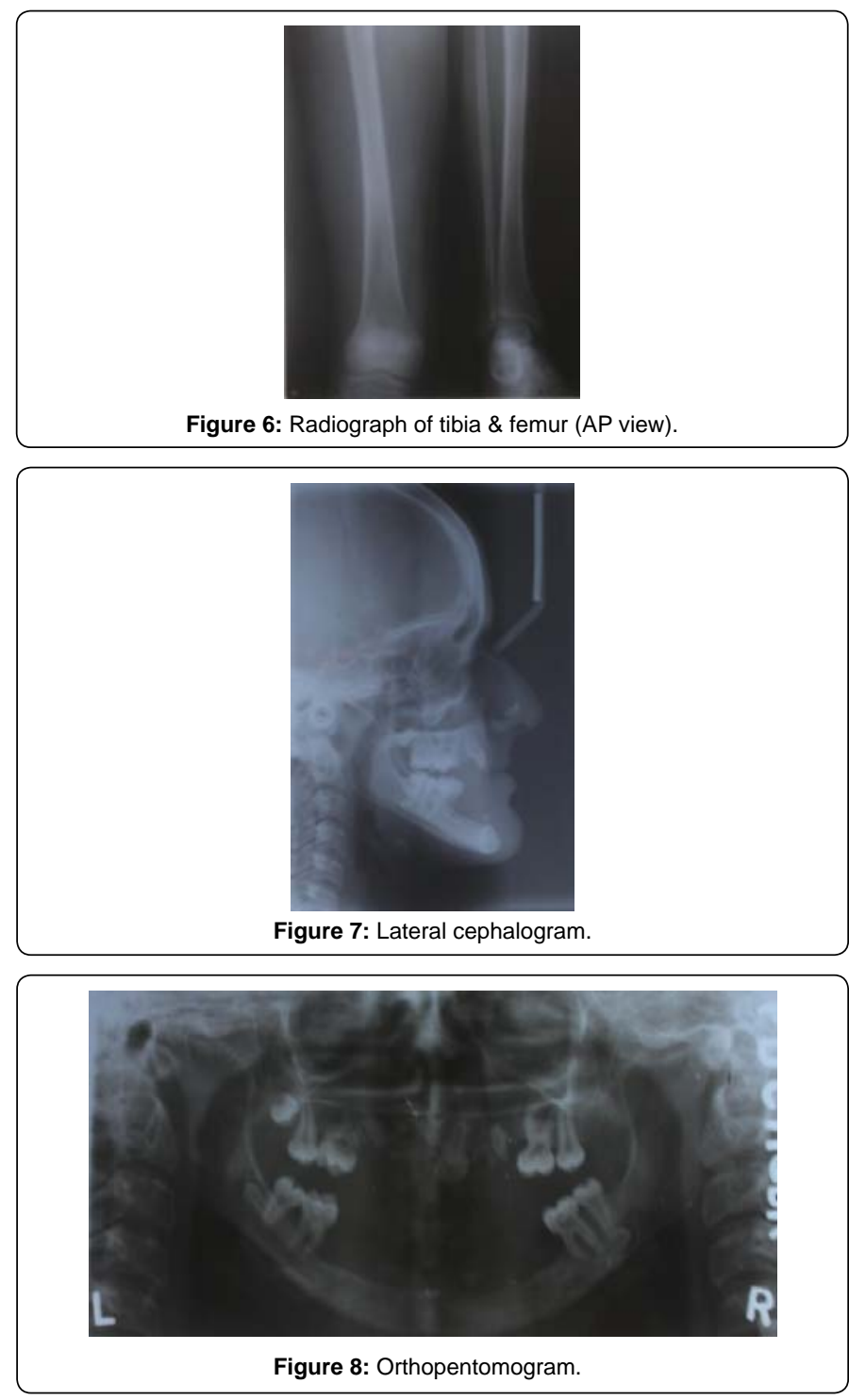

third quadrant. Second molars of first, third and fourth quadrants were carious.

Radiographic investigations included extra-oral radiographs of the Chest (AP view) (Figure 5), tibia and femur (AP view) (Figure 6) were done for eliciting signs of hypocalcification and bowing of legs respectively. But, no signs of any abnormalities were observed.

A lateral cephalogram also was done which was also non contributory (Figure 7). An Orthopentomogram was taken and it revealed following features (Figure 8).

The second molar and the central incisor of the first quadrant, second molar of second quadrant and second molars of third and fourth quadrant had wide pulp chambers and root canals with reduced bone height. Retained roots of deciduous maxillary right first molar, maxillary left canine and mandibular right lateral incisor were seen. The radiograph also revealed mandibular right and left second premolar to be impacted.

A complete blood investigation was done but the results were within normal range.

The biochemical analysis for alkaline phosphatase levels were also seen to be within the normal limits. 


\section{Discussion}

Hypophosphatasia is an uncommon inherited disorder with an incidence of $1: 100,000^{6}$. It is manifested by skeletal demineralization, decrease in activity of bone, kidney, serum tissue non-specific alkaline phosphatase due to mutations in gene encoding tissue non-specific alkaline phosphatase [7].

Alkaline phosphatase catalyzes formation of matrix for bones and cementum, and its reduction results in hypoplasia and defective mineralization of alveolar bone and cementum [8].

Inadequate formation of dentin, cementum and bone is the most likely explanation of dental abnormalities [9].

The case described in this article showed the alkaline phosphatase levels to be normal when the patient reported for dental treatment at the age of 15 years. The dental literature also shows that the levels of alkaline phosphatase may approach normal as they grow older [10].

The present case also showed that the deciduous as well as the permanent dentition to be affected. This was in concurrence with the report by Olsson et al. [2] who have shown hypophosphatasia to affect deciduous as well as permanent dentition.

This case showed loss of deciduous and permanent teeth, wide pulp chambers in some teeth, presence of dental caries, gingival recession and reduced alveolar bone height which was in accordance with Herasse et al. [5] who also showed unexplained premature loss of teeth, dental caries, wide pulp chambers and reduced alveolar bone height.

\section{Conclusion}

Hypophosphatasia is an inherited disorder, can occur due to the mutations in tissue non-specific alkaline phosphatase gene. It has a wide spectrum of skeletal and dental presentation. Dental manifestations in hypophosphatasia appear to be the commonest and sometime the only feature. Such a condition when encountered is known as odontohypophosphatasia.

The path to correct diagnosis may be complicated by the fact that odontohypophosphatasia may not be accompanied by biochemical abnormalities. Hence, diagnosis of odontohypophosphatasia should also be considered in cases of unexplained exfoliation of deciduous and permanent teeth.

\section{Rerefences}

1. Lam ACF, Lam CW, Tang MHY, Chu JWY, Lam STS (2006) A case of perinata lethal form of hypophosphatasia; and review of literatures. HK J Paediatr 11: 341-346.

2. Olsson A, Matsson L, Blomquist HK, Larsson A, Sjodin B (1996) Hypophosphatasia affecting permanent dentition. J Oral Pathol Med 25: 343347

3. Watanabe H, Goseki-Sone M, limura T, Oida S, Orimo H, et al. (1999) Molecular diagnosis of hypophosphatasia with severe periodontitis. J Periodontol 70: 688691.

4. Iqbal SJ, Plaha DS, Linforth GH, Dalglerish R (1999) Hypophosphatasia: diagnostic application of linked DNA markers in the dominantly inherited adult form. Clin Sci 97: 73-78.

5. Herasse M, Spentchian M, Taillandier A, Keppler-Noreuil K, Fliorito AN et al. (2003) Molecular study of three cases of odontohypophosphatasia resulting from heterozygosity for mutations in the tissue non-specific alkaline phosphatase gene. J Med Genet 40: 605-609.

6. Kritsaneepaiboon S, Jaruratanasirikul S, Dissaneevate $S$ (2006) Clinics in diagnostic imaging (112). Perinatal lethal hypophosphatasia (PLH). Med J 47: 987-993.

7. Etienne M (2007) Hypophosphatasia. Orphanet Journal of Rare 2: 1-7.

8. Watanabe H, Umeda M, Seki T, Ishikawa I (1993) Clinical and laboratory studies of severe periodontal disease in an adolescent associated with hypophosphatasia. A case report. J Periodontol 64: 174-180.

9. Macfarlane JD, Swart JG (1989) Dental aspects of hypophosphatasia: A case report, family study, and literature review. Oral Surg Oral Med Oral Pathol 67: 521-526.

10. Neville BW, Damm DD, Allen CM, Bouquot JE (2008) Oral and maxillofacia pathology. Philadelphia: Saunders 984. 